Keywords: nasopharyngeal carcinoma; local recurrence; prognostic-score model; intensity-modulated radiotherapy (IMRT); late complications

\title{
Prognostic model for survival of local recurrent nasopharyngeal carcinoma with intensity-modulated radiotherapy
}

\author{
Y-M Tian ${ }^{1,4}$, Y-H Tian ${ }^{2,3,4}$, L Zeng ${ }^{1,4}$, S Liu ${ }^{1}$, Y Guan ${ }^{1}$, T-X Lu ${ }^{1}$ and F Han ${ }^{*, 1}$ \\ ${ }^{1}$ Department of Radiation Oncology, Sun Yat-sen University Cancer Center, State Key Laboratory of Oncology in South China, \\ Collaborative Innovation Center for Cancer Medicine, Guangzhou, Guangdong Province, People's Republic of China; ${ }^{2}$ Cancer \\ Research Institute, Southern Medical University, Guangzhou, Guangdong Province, People's Republic of China and ${ }^{3}$ Department \\ of Oncology, Armed Police Hospital of Guangdong Province, Guangzhou, Guangdong Province, People's Republic of China
}

Background: Intensity-modulated radiotherapy (IMRT) is the main salvage treatment for advanced locally recurrent nasopharyngeal carcinoma (NPC); however, survival outcomes vary. We aimed to construct a prognostic-score model to identify patients who could benefit from salvage IMRT.

Methods: This retrospective study involved 251 patients with locally recurrent NPC. The following parameters were analysed following IMRT: patient performance status, age, gender, late complications, T-stage of recurrence, synchronous nodal recurrence, primary gross tumour volume (GTV-nx), disease-free interval, re-irradiation dose and chemotherapy. The model was based on the hazard ratio coefficients of six significantly negative prognostic factors for survival.

Results: Significantly negative prognostic factors included Karnofsky Performance Status $\leqslant 70$, age $>50$ years, late complications, recurrent $T_{3-4}$ stage, synchronous nodal recurrence and GTV-nx $>30 \mathrm{~cm}^{3}$. Three subgroups were defined according to model scores: low risk (0-4), intermediate risk (5-8) and high risk (9-15). The 5-year overall survival rates were $64.3 \%, 32.2 \%$ and $7.7 \%$, respectively. The main cause of death was radiation-induced complications.

Conclusion: The prognostic-score model demonstrated that re-irradiation with IMRT is suitable for low-risk and intermediate-risk patients but may be unsuitable for high-risk patients. Further research into the protection of critical adjacent organs to reduce late complications in these patients is warranted.

Nasopharyngeal carcinoma (NPC) is common in southern China, especially in the Guangdong province, where rates range from 20 to 30 cases per 100000 population (Yu and Yuan, 2002; Wei and Sham, 2005). Radiotherapy is the standard treatment for early disease, and combined radiotherapy and chemotherapy is applied for advanced disease (Al-Sarraf et al, 1998; Wee et al, 2005). Despite significant improvements in local control due to advances in radiotherapy and combined modality treatments, local recurrence remains a major cause of treatment failure in patients with advanced NPC (Kam et al, 2004; Lee et al, 2005). However, a significant proportion of patients can achieve long-term survival following salvage therapy; therefore, aggressive treatment with curative intent is usually recommended (Lee et al, 1993; Yu et al, 2005). Owing to the high risk of infiltration, most cases of recurrent NPC are already extensive at diagnosis, and external beam radiation therapy is often the only selection for salvage treatment. Conventional radiotherapy can be difficult, with a high incidence of late complications due to the constraints of critical structures in the vicinity of the target tumour, which would have already received a high dose of radiation during the first course

\footnotetext{
*Correspondence: Dr F Han; E-mail: gzhanfei@163.com
}

${ }^{4}$ These authors contributed equally to this work.

Received 2 August 2013; revised 12 October 2013; accepted 21 October 2013; published online 12 December 2013 
of radiotherapy. As such, the 5-year overall survival (OS) rate is poor, with a range of only $8-36 \%$ (Teo et al, 1998; Lee et al, 2000; Zheng et al, 2005). The introduction of intensity-modulated radiotherapy (IMRT) can provide a more favourable balance between target coverage and the sparing of adjacent organs (Hsiung et al, 2002). Preliminary reports have demonstrated that better disease control and patient survival can be achieved with a relatively low incidence of late complications by using IMRT in patients with locally recurrent NPC (Lu et al, 2004; Chua et al, 2005; Roeder et al, 2011; Han et al, 2012; Hua et al, 2012; Qiu et al, 2012).

Along with other solid tumours, locally recurrent NPC is a highly heterogeneous disease (Lee et al, 2000; Hua et al, 2012), and patient survival following IMRT varies, ranging from several months to long-term survival. Therefore, developing a model to predict the survival outcomes in patients with locally advanced NPC could facilitate the identification of those patients who would most benefit from IMRT. Such a model could also contribute to the design of clinical trials for locally recurrent NPC. The aim of this study was to design a prognostic-score model for this purpose through the retrospective investigation of prognostic factors and outcomes in patients with locally recurrent NPC following IMRT.

\section{MATERIALS AND METHODS}

Patient selection and pretreatment evaluation. A total of 251 patients with locally recurrent NPC were diagnosed and received re-irradiation using IMRT at the Sun Yat-Sen University Cancer Centre between January 2001 and May 2009. Local recurrence was confirmed by biopsy for most of the patients. Those patients with recurrence in inaccessible sites, such as the skull base and intracranial cavity, were diagnosed radiologically according to local disease progression.

The pretreatment evaluation involved a complete medical history, and physical examinations included the patient's performance status and any severe complications (i.e., trismus, radiation encephalopathy, cranial nerve palsy and mucosa necrosis). A complete blood count, renal and liver function tests and nasopharyngoscopy were required. Magnetic resonance imaging (MRI) or computed tomography (CT) of the nasopharynx and neck was performed for the staging evaluations. Chest X-rays, abdominal sonography and bone scans were performed to detect distant metastases. Positron emission tomography-CT (PET-CT) was performed at the physician's discretion. All patients with recurrent NPC were re-staged according to the classification system of the American Joint Committee on Cancer (AJCC; 2002).

Intensity-modulated radiotherapy. Target volumes were delineated according to our institution's treatment protocol, in agreement with the International Commission on Radiation Units and Measurements (ICRU) reports 50 and 62. The gross tumour volumes (GTVs) at the primary site (GTV-nx) and neck (GTV-nd) included the total disease volumes visualised using CT or MRI. The clinical target volumes (CTVs) were designed to encompass microscopic disease by including the GTV plus a $1-$ to $1.5-\mathrm{cm}$ margin; smaller margins $(<3 \mathrm{~mm})$ were applied near critical intracranial structures or the spinal cord. The CTV also included the entire nasopharynx and lymph node-positive regions. No elective re-irradiation of the uninvolved regional lymph nodes was performed (Figure 1). Additional 2- to 3-mm margins were added to the CTV and GTV to create the planning target volume (PTV) for setup variability and internal motion. The organs at risk (OARs) included the brainstem, spinal cord, optic nerves, optic chiasm, pituitary gland, lens, temporal lobes, parotid glands, temporomandibular joints and mandible. The dose constraints to the OARs were limited by the threshold doses, the disease-free interval (DFI) after primary radiotherapy and the patient's general condition. For example, patients with severe complications, poor status and/or diabetes were prescribed a relatively low dose of $60 \mathrm{~Gy}$ to the GTV, and more attention was given to sparing the adjacent organs.

The prescribed doses were 60-70 Gy to the GTV and 50-54 Gy to the CTV in 27-35 fractions. All patients received full-course IMRT with $6 \mathrm{MV} \mathrm{X}$-rays generated using a Clinac-600C linear accelerator (Varian Medical Systems, Palo Alto, CA, USA).

Chemotherapy. Cisplatin-based induction or concurrent chemotherapy was administered in 126 patients with $\mathrm{rT}_{3-4}$ and/ or bulky gross tumours. The groups included 46 patients with concurrent chemoradiotherapy, 67 patients with induction chemotherapy followed by radiotherapy and 13 patients with induction and concurrent chemotherapy.

Patient assessment and follow-up. After treatment completion, patients were evaluated at least once every 3 months during the first 3 years and then every 6 months thereafter until death. Magnetic resonance imaging or CT of the head and neck, chest $\mathrm{X}$-ray radiography and abdominal ultrasonography were performed annually. Severe radiation toxicities secondary to treatment were assessed and scored according to the radiation morbidity scoring criteria of the Radiation Therapy Oncology Group (RTOG).

Statistical analysis. Overall survival, local-regional failure-free survival (LRFFS) and distant failure-free survival (DFFS) were calculated using the Kaplan-Meier method. Differences between survival curves were compared using the log-rank test. Univariate and multivariate analyses were performed using the Cox proportional hazards model. The time periods were calculated from the date when recurrence was diagnosed to the date of each event or the last follow-up. Disease-free interval was defined as the time between the completion of the primary radiotherapy to the diagnosis of recurrence in patients who had achieved a complete response. The following factors were included in the analyses: patients' characteristics (performance status, age, gender and late complications); disease characteristics (T-stage of recurrence, synchronous nodal recurrence, GTV-nx and DFI); and treatment methods (chemotherapy, re-irradiation dose). A $P$-value $<0.05$ was considered significant.

The regression coefficient $(\beta)$ for each independent prognostic factor was derived from the Cox regression equation $\left(H R=e^{\beta}\right)$, where HR is the hazard ratio. This value was then converted into an integer to provide a score index for the prognostic-score model.

\section{RESULTS}

Clinicopathological characteristics. The clinicopathological characteristics of the patients are given in Table 1 . The median age was 45 years (range, 21-75 years). Approximately 39 out of 251 (15.5\%) patients presented with late complications after the first course of radiotherapy, including 16 patients with trismus, 10 with radiation encephalopathy, 9 with cranial nerve palsy and 4 with mucosa necrosis (xerostomia and hearing loss were not included).

Treatment planning and dosimetry. The median minimum, mean and maximum doses for the GTV-nx were 57.5 Gy (range, 33.3-70.5 Gy), 70.7 Gy (range, 61.1-79.7 Gy) and 76.7 Gy (range, $65.5-85.8 \mathrm{~Gy}$ ), respectively. The median percentage of the GTV receiving $95 \%$ of the prescribed dose (V95) was 98.1\% (range, 56.1-100\%); the median dose encompassing $95 \%$ of the GTV-nx (D95) was 68.1 Gy (range, 64.5-71.4 Gy).

Patient survival outcomes. The median follow-up period was 40.0 months (range, 3.0-147.0 months). A total of 15 patients were lost to follow-up between 6.0 and 61.0 months. Local-regional failure 

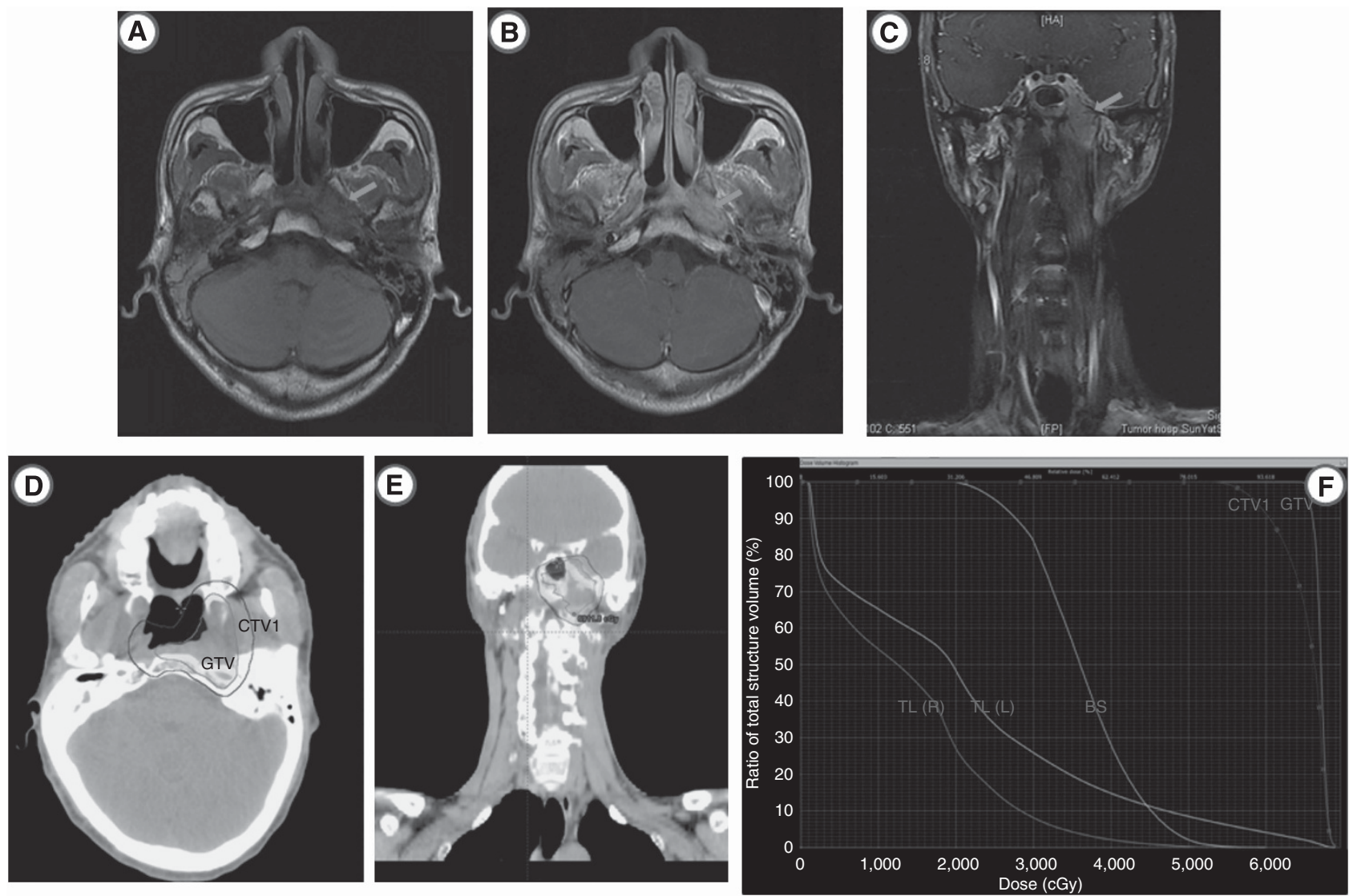

Figure 1. Representative MR images and treatment plan of one patients. Axial T1-weighted (A), contrast-enhanced T1-weighted (B), and coronal contrast-enhanced fat-saturated T1-weighted MR images (C) show a left-sided recurrent NPC with lateral extension to parapharygneal space and superior extension into the left intracranial cavity. (D-F) Delineation of target volume and dose-volume histogram (DVH) included temporal lobe $(\mathrm{TL})$ and brainstem (BS).

was confirmed in 48 out of 251 (19.1\%) patients, including 43 out of $48(89.6 \%)$ with local failure and 5 out of $48(10.4 \%)$ with regional failure. Distant metastases occurred in 37 out of 251 (14.7\%) patients, including five patients with local failure. The 3 -year and 5-year LRFFS rates were $80.6 \%$ and $75.1 \%$, respectively. The 3-year and 5-year DFFS rates were 86.1 and $80.6 \%$, respectively.

A total of 159 out of 251 (63.3\%) patients died during this study. Of these, 77 out of 159 (48.4\%) deaths were due to radiationinduced injuries, including 41 out of 77 (53.2\%) from mucosa necrosis or massive haemorrhage, 14 out of 77 (18.2\%) from radiation encephalopathy and 22 out of 77 (28.6\%) from other radiation-related injuries. In addition, 37 out of 159 (23.3\%) patients died due to local-regional failures and 32 out of 159 (20.1\%) due to distant failures. Other causes responsible for 15 out of $159(9.4 \%)$ deaths included 3 out of $15(20.0 \%)$ cases of cardiac disease, 2 out of $15(13.3 \%)$ cases of leukaemia, 2 out of $15(13.3 \%)$ cases of intracranial infection, 1 out of $15(6.7 \%)$ case of digestive diseases and 5 out of $15(33.3 \%)$ unknown causes. The 3 -year and 5 -year OS rates were $53.2 \%$ and $41.1 \%$, respectively.

Late complications. Treatment was not interrupted due to severe acute toxicity in any of the patients. However, most patients developed mild to moderate acute toxicities, including mucositis, xerostomia and otitis media. Approximately 20 out of 251 (8.0\%) patients experienced grade- 3 acute mucositis, 18 of who underwent concurrent chemoradiotherapy. After the completion of IMRT, 84 out of $251(33.4 \%)$ patients developed mucosa necrosis, 61 out of 251 (24.3\%) developed temporal lobe necrosis and 19 out of 251 (7.6\%) developed cranial neuropathy during the follow-up period.
Significant xerostomia, hearing loss and trismus were common, and 102 out of $251(40.6 \%)$ patients developed at least one of these conditions. Those patients with $\mathrm{rT}_{3-4}$ tumours or GTV-nx $>30 \mathrm{~cm}^{3}$ exhibited significantly higher incidences of mucosa necrosis than patients with $\mathrm{rT}_{1-2}$ stage tumours (36.8 vs $26.1 \%$; $P=0.04)$ or with GTV-nx $\leqslant 30 \mathrm{~cm}^{3}$ (38.7 vs $\left.23.0 \% ; P<0.01\right)$.

Univariate analyses. The statistically significant negative prognostic factors for OS were Karnofsky Performance Status (KPS) $\leqslant 70$, age $>50$ years, severe late complications, $\mathrm{rT}_{3-4}$ tumours, GTV-nx $>30 \mathrm{~cm}^{3}$ and mean re-irradiation dose $>68.0 \mathrm{~Gy}$. Synchronous nodal recurrence was marginally significant in predicting poor OS. These results, including $P$-values, are summarised in Table 2. The statistically significant negative prognostic factors for LRFFS were KPS $\leqslant 70$ (HR: 3.64; $P<0.01$ ), $\mathrm{rT}_{3-4}$ tumours (HR: 3.68; $P<0.01$ ) and DFI $<24$ months (HR: 2.14; $P=0.01$ ). The only significant negative prognostic factor for DFFS was recurrence of $\mathrm{rT}_{3-4}$ tumours (HR: $1.53 ; P=0.03$ ).

Multivariate analyses. The significant independent negative prognostic factors for OS were KPS $\leqslant 70$, age $>50$ years, severe late complications, $\mathrm{rT}_{3-4}$ tumours, synchronous nodal recurrence and GTV-nx $>30 \mathrm{~cm}^{3}$. These results, including $P$-values, are summarised in Table 3. The survival curves of patients according to the different prognostic factors are shown in Figures 2 and 3. Significant independent negative prognostic factors for LRFFS were KPS $\leqslant 70$ (HR: 3.06; $P=0.04$ ) and $\mathrm{rT}_{3-4}$ tumours (HR: 5.46; $P=0.02$ ). However, no significant independent factor was associated with DFFS by multivariate analysis. 


\begin{tabular}{|c|c|}
\hline Characteristic & $\mathbf{N}(\%)$ \\
\hline \multicolumn{2}{|c|}{ Karnosky performance score } \\
\hline$>70$ & $238(94.8)$ \\
\hline$\leqslant 70$ & $13(5.2)$ \\
\hline \multicolumn{2}{|l|}{ Gender } \\
\hline Male & $195(77.7)$ \\
\hline Female & $56(22.3)$ \\
\hline \multicolumn{2}{|l|}{ Age (year) } \\
\hline Median & 45 \\
\hline Range & $21-75$ \\
\hline \multicolumn{2}{|l|}{ Pathology } \\
\hline WHO I & $5(2.0)$ \\
\hline WHO II-III & $196(78.0)$ \\
\hline Imaging findings only & $50(20.0)$ \\
\hline \multicolumn{2}{|c|}{ Presence of significant complications } \\
\hline No & $212(84.5)$ \\
\hline Yes & $39(15.5)$ \\
\hline \multicolumn{2}{|l|}{ DFI (month) } \\
\hline$>24$ & $133(53.0)$ \\
\hline$\leqslant 24$ & $118(47.0)$ \\
\hline \multicolumn{2}{|c|}{ rT stage (2002AJCC) } \\
\hline T1 & $22(8.8)$ \\
\hline T2 & $31(12.4)$ \\
\hline T3 & $90(35.9)$ \\
\hline T4 & $108(43.0)$ \\
\hline \multicolumn{2}{|c|}{ Presence of synchronous nodal recurrence } \\
\hline No & $212(84.5)$ \\
\hline Yes & $39(15.5)$ \\
\hline \multicolumn{2}{|c|}{ Volume of GTV-nx $\left(\mathrm{cm}^{3}\right)$} \\
\hline$\leqslant 30$ & $100(39.8)$ \\
\hline$>30$ & $151(60.2)$ \\
\hline \multicolumn{2}{|c|}{ Prior radiation dose (Gy) } \\
\hline Median & 70 \\
\hline Range & $64-82$ \\
\hline \multicolumn{2}{|l|}{ Chemotherapy } \\
\hline Yes & $126(50.2)$ \\
\hline No & $125(49.8)$ \\
\hline \multicolumn{2}{|c|}{$\begin{array}{l}\text { Abbreviations: } D F I=\text { disease-free interval; } G T V=\text { gross tumour volume; } K P S=\text { Karnosky } \\
\text { performance score. } \\
D F I \text {, interval time from the end of first course of radiotherapy to recurrence at diagnosis. }\end{array}$} \\
\hline
\end{tabular}

Scoring of patients according to the prognostic-score model. The prognostic-score model was based on the regression coefficients of the six significantly independent negative prognostic variables, as described above. A score of 2 or 3 was assigned for each factor, according to the HR ( $n$ ) value (Table 4). If a factor was not independently significant, a score of 0 was recorded. The maximum possible score for each patient was 15 . The prognostic score for each of the 251 patients was calculated from the sum of the individual scores. Patients were then assigned to three risk
Table 2. Univariate analysis of variables correlated with overall survival

\begin{tabular}{|c|c|c|c|}
\hline Characteristic & $\begin{array}{c}\text { 5-year OS } \\
(\%)\end{array}$ & $\operatorname{HR}(95 \% \mathrm{Cl})$ & $P$-value \\
\hline $\mathrm{KPS},>70 / \leqslant 70$ & $42.3 / 14.4$ & $2.65(1.50-4.67)$ & $<0.01^{a}$ \\
\hline Gender, male/female & $41.5 / 39.8$ & $1.13(0.79-1.63)$ & 0.48 \\
\hline Age (year), $\leqslant 50 />50$ & $43.3 / 37.5$ & $1.48(1.08-2.03)$ & $0.02^{a}$ \\
\hline $\begin{array}{l}\text { Significant complications, } \\
\text { no/yes }\end{array}$ & $46.1 / 15.4$ & $2.36(1.60-3.47)$ & $<0.01^{\mathrm{a}}$ \\
\hline DFI (month), $>24 / \leqslant 24$ & $42.7 / 39.5$ & $1.05(0.77-1.43)$ & 0.73 \\
\hline \multicolumn{4}{|l|}{$r T$} \\
\hline rT1 & 81.8 & Baseline & \\
\hline rT2 & 64.5 & $2.50(0.67-6.64)$ & 0.22 \\
\hline rT3-4 & 32.4 & $3.00(1.89-4.7)$ & $<0.01^{\mathrm{a}}$ \\
\hline $\begin{array}{l}\text { Synchronous nodal recurrence, } \\
\text { no/yes }\end{array}$ & $43.0 / 31.7$ & $1.43(0.98-2.11)$ & 0.06 \\
\hline $\begin{array}{l}\text { Volume of GTV-nx }\left(\mathrm{cm}^{3}\right) \\
\leqslant 30 />30\end{array}$ & $59.9 / 26.9$ & $1.52(1.30-1.97)$ & $<0.01^{\mathrm{a}}$ \\
\hline Chemotherapy, yes/no & $35.4 / 47.0$ & $1.31(0.96-1.79)$ & 0.09 \\
\hline $\begin{array}{l}\text { Re-irradiation dose (Gy), } \\
\leqslant 68 />68\end{array}$ & $48.9 / 36.9$ & $1.50(1.05-2.14)$ & $0.02^{\mathrm{a}}$ \\
\hline \multicolumn{4}{|c|}{ 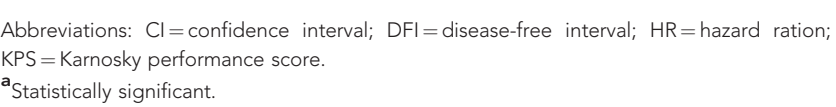 } \\
\hline
\end{tabular}

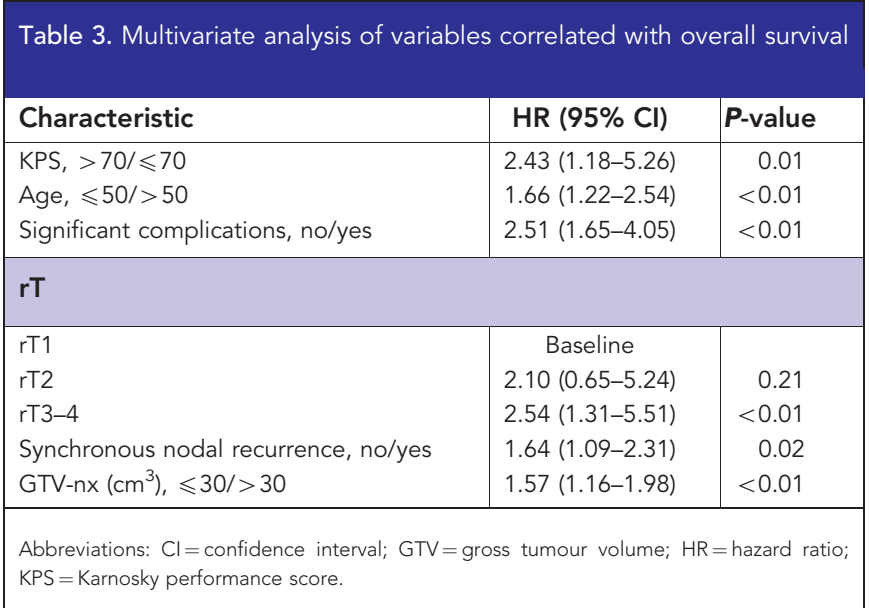

stratification groups, based on the prognostic scoring system: (I) the low-risk group (total score, $0-4$ ) included 79 out of 251 (31.5\%) patients; (II) the intermediate-risk group (total score, 5-8) included 146 out of 251 (58.2\%) patients; and (III) the high-risk group (total score, 9-15) included 26 out of 251 (10.6\%) patients. The median survival periods of the patients in these groups were 62.0, 25.5 and 8.5 months, respectively $(P<0.001$; Figure 4$)$. The 5 -year OS rates were $64.3 \%, 32.2 \%$ and $7.7 \%$, respectively. The survival curves were distinctly separated between these groups (Figure 3D).

\section{DISCUSSION}

In the management of locally recurrent NPC, aggressive treatment, including surgery or re-irradiation, instead of chemotherapy alone is usually recommended to improve the chance of long-term survival (Lee et al, 1993; Yu et al, 2005). Chemotherapy is considered a palliative treatment for advanced recurrence, which is 

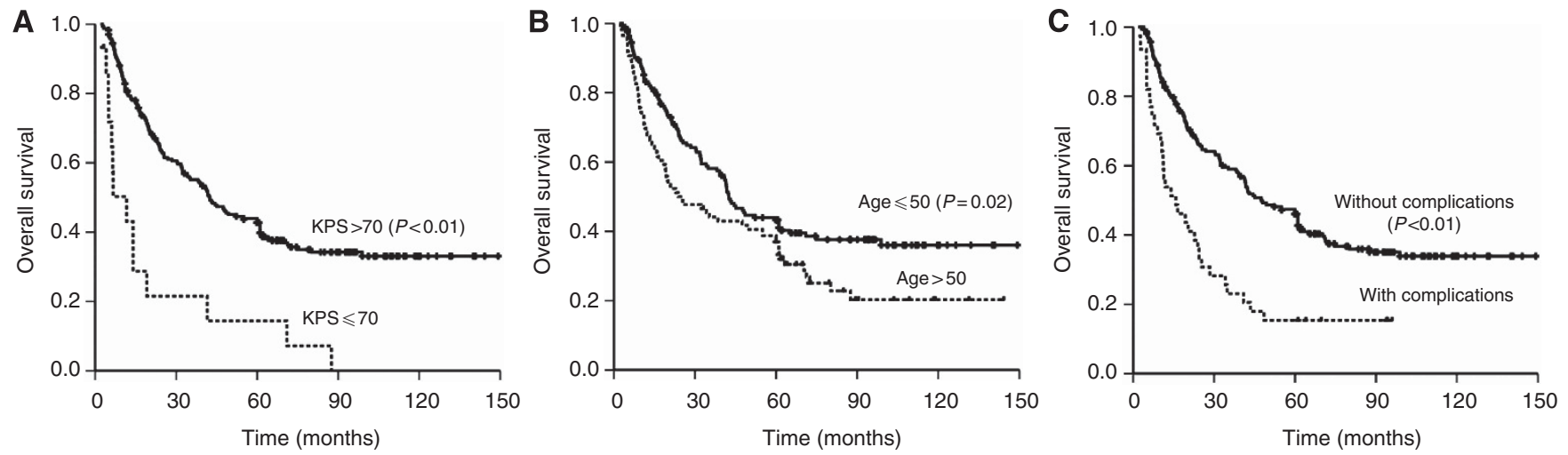

Figure 2. Kaplan-Meier survival curves for OS according to KPS (A), age (B) and significant complications (C).

A
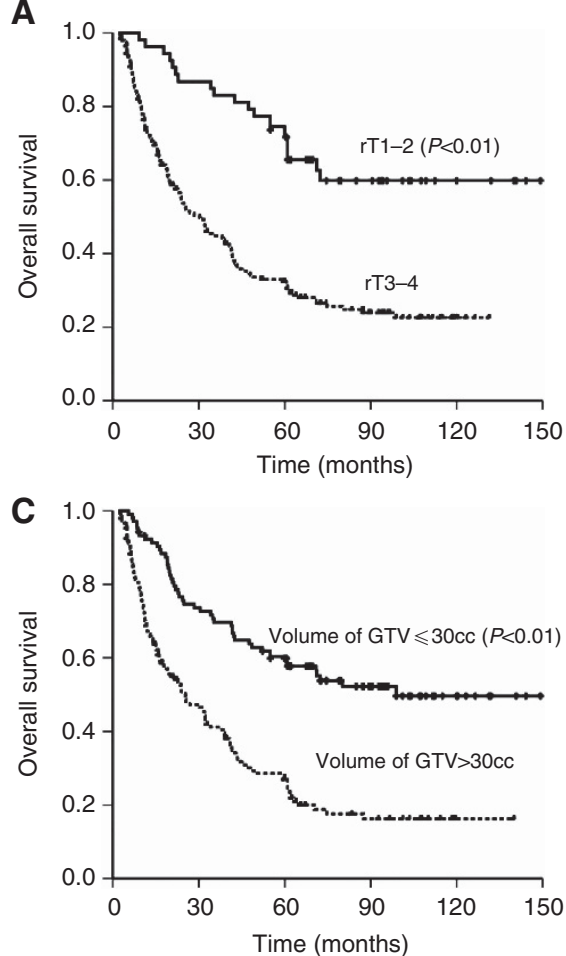

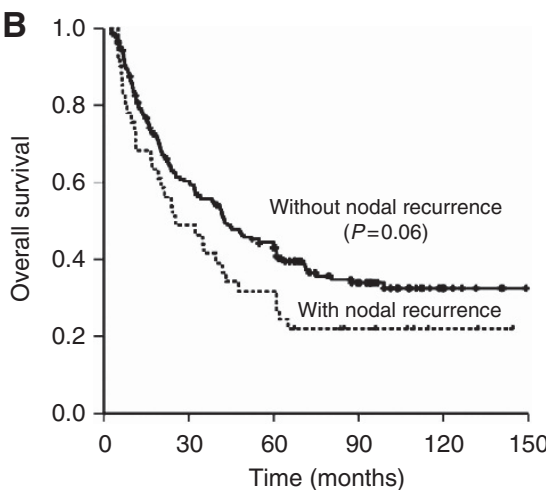

D

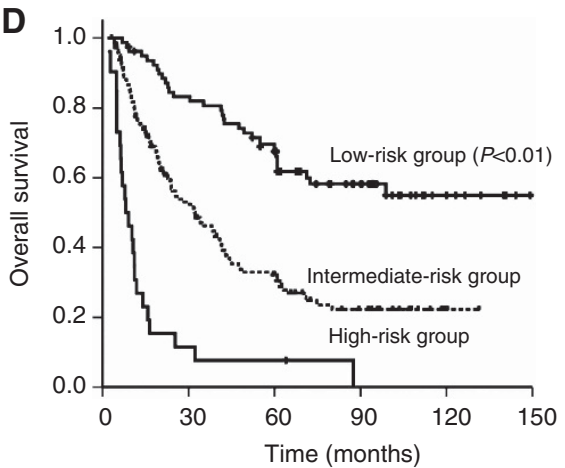

Figure 3. Kaplan-Meier survival curves for OS according to recurrent stage (A), presence of synchronous nodal recurrence (B), volume of GTV-nx (C) and the different risk groups (D).

\section{Table 4. Prognostic index score according to the HR ( $n$ value)}

\begin{tabular}{|l|c|c|}
\hline Characteristic & Score & $\boldsymbol{n}\left(\mathbf{H R}=\mathbf{e}^{\boldsymbol{n}}\right)$ \\
\hline Volume of GTV-nx $>30 \mathrm{~cm}^{3}$ & 2 & 0.45 \\
\hline Synchronous nodal recurrence & 2 & 0.49 \\
\hline Age $>50$ years & 2 & 0.51 \\
\hline KPS $\leqslant 70$ & 3 & 0.89 \\
\hline With significant complications & 3 & 0.92 \\
\hline Recurrent T3-4 & 3 & 0.93 \\
\hline Maximum score & 15 & \\
\hline $\begin{array}{l}\text { Abbreviations: GTV = gross tumour volume; HR= hazard ratio; KPS = Karnosky performance } \\
\text { score. } \\
\text { According to the HR ( } n \text { value), the maximum value of recurrent T3-4 is } 1.5 \text { times of the } \\
\text { minimum value of volume of GTV-nx. To simplicity, we assume that the value of baseline is } 2 .\end{array}$ \\
\hline
\end{tabular}

not suitable for local aggressive therapies or patients with distant metastases (Oksuz et al, 2004). Salvage surgical resection in patients with early-stage recurrence has been shown to provide satisfactory disease control and improved quality of life (Wei, 2000; Hsu et al, 2001). Brachytherapy and stereotactic radiotherapy have also been used successfully in patients with NPC but are limited by the extension and volume of the disease (Law et al, 2002; Chua et al, 2006). Hsiung et al (2002) demonstrated the superior dose distribution and sparing of normal tissues with IMRT compared with three-dimensional conformal radiotherapy as a boost or salvage treatment for locally recurrent NPC. In a study by Lu et al (2004), 100\% loco-regional control (LRC) was achieved with IMRT in 49 patients without any severe late complications after a median follow-up period of 9 months. Chua et al (2005) reported a 1-year LRC rate of $56 \%$ and an OS rate of $63 \%$ in 31 patients following IMRT. In this study, we found similar 5-year LRFFS and OS rates of $75.1 \%$ and $41.1 \%$, respectively, supporting these previous reports 


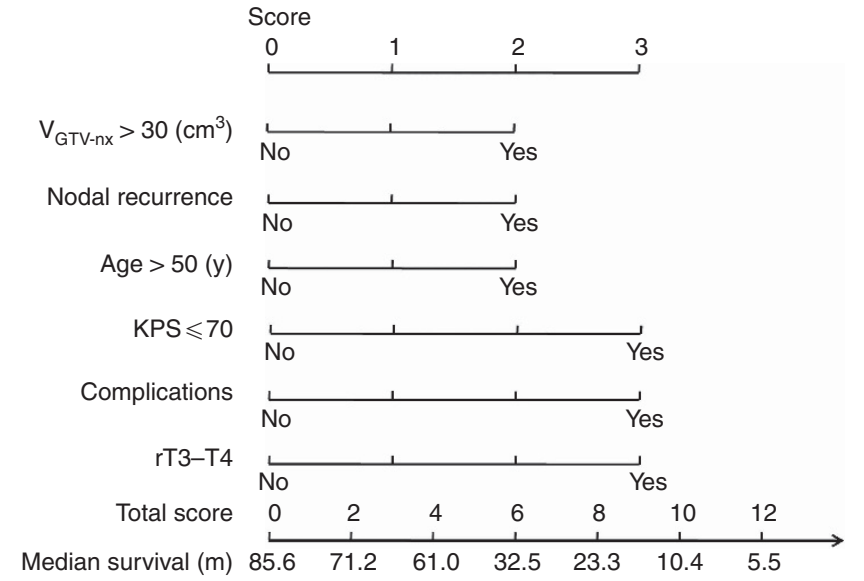

Figure 4. A prognostic model was built based on six patient and disease factors. The total score can be calculated in predicting the median survival according to the prognostic factors.

that IMRT can be an effective salvage treatment in patients with locally recurrent NPC.

Poor performance status is known to be associated with old age, and advanced disease and a high incidence of severe complications often indicate that these patients are unable to tolerate high-dose IMRT and chemotherapy, resulting in poor survival and little or no treatment benefit. A meta-analysis of head and neck cancers found that the benefits of chemoradiotherapy decreased with increasing age and that the proportion of deaths unrelated to the cancer significantly increased with age (Pignon et al, 2009). Although age is accepted as a significant independent variable in the survival of patients with head and neck cancer (including primary NPC), the cutoff value reported in different studies has been inconsistent (Chow et al, 2002; Corry et al, 2006). In agreement with an earlier study (Oksuz et al, 2004), our results demonstrated that the patient performance status and age have prognostic significance. The 5 -year OS rate in patients $>50$ years was only $37.5 \%$, compared with $43.3 \%$ in younger patients.

Complications before retreatment associated with the poor survival of patients with local recurrent NPC include trismus, temporal lobe necrosis, cranial neuropathy (e.g., neuropathy of cranial nerves IX-XII) and mucosa necrosis. These conditions not only affect quality of life but can lead to poor status or death, and they became more severe after re-irradiation. In this study, the 5 -year OS rate of patients with severe late complications was only $15.4 \%$ and the HR was 2.53 . These findings indicate that a patient's characteristics, including performance status, age and severe late complications, should be important considerations in planning salvage treatments using IMRT for local failure in NPC.

The prognostic factors that have been most consistently reported for local control and OS are disease characteristics, such as the T-stage of recurrence and GTV-nx (Chua et al, 2005; Roeder et al, 2011; Han et al, 2012; Hua et al, 2012; Qiu et al, 2012). Patients with advanced-stage tumours and GTV-nx $>30 \mathrm{~cm}^{3}$ generally experienced poor tumour control and shorter survival times. These outcomes may be due to the following reasons: suboptimal dose distribution in advanced and extensive disease is compromised by the protection of critical, adjacent structures; poor blood supply and hypoxia associated with bulky tumours and tissue fibrosis can lead to decreased radiation and/or chemotherapy sensitivity; and there is a high incidence of necrosis and/or massive haemorrhage in advanced and bulky disease. In clinical practice, salvage treatments for advanced and bulky disease are challenging and require a balance between the benefits of aggressive treatment and potentially fatal complications. In this study, synchronous nodal recurrence was related to poor survival, as these patients are more prone to distant metastases, with a 5-year DFFS rate of $69.2 \%$, compared with $82.1 \%$ in patients with local recurrence alone. However, the difference was not significant.

The heterogeneity of locally recurrent NPC indicates that not all patients will benefit from re-irradiation using IMRT, as they may experience poor disease control and severe late complications. Therefore, we constructed a prognostic-score model based on the six most significantly negative prognostic factors identified in patients following IMRT, as described above. This model was used to stratify the patients into three subgroups according to low-, intermediate- and high-risk scores. The aim was to discriminate between patients with good and poor prognoses and to select candidates more suitable for re-irradiation using IMRT. The patients in the low- and intermediate-risk groups achieved good disease control and survival after IMRT, with median survival periods of 62.0 months and 25.5 months, respectively, indicating that re-irradiation using IMRT can be a suitable option. However, the median survival of patients in the high-risk group was only 8.5 months. Some of the high-risk patients were unlikely to benefit from high-dose re-irradiation due to poor disease control and severe late complications. Therefore, further investigations to establish effective treatment strategies, including chemotherapy and targeted therapy, for these high-risk patients are warranted.

Although excellent disease control can be achieved with highdose irradiation using IMRT, the benefits need to be balanced against the risk of severe late complications, which remains the main cause of death following treatment. Mucosa necrosis and massive haemorrhage are among the main challenges, and their incidences are related to individual patient tolerance and radiation dose (Marx, 1983; Hua et al, 2009). Furthermore, patients with advanced or bulky disease experience significantly higher rates of mucosa necrosis. Other main causes of death include temporal lobe necrosis and poor medical conditions related to radiation exposure, such as cranial nerve IX-XII palsy. To reduce the risk of late complications, investigations to determine the optimal total dose and dose-fractions for disease control, while sparing adjacent critical organs, are currently being conducted in our centre.

The purpose of this prognostic-score model was to enable those patients who would most benefit from IMRT to be identified and to facilitate improvements in the therapeutic ratio of re-irradiation in NPC. Our findings demonstrated that re-irradiation using IMRT can be an appropriate choice for patients in the low- and intermediate-risk groups but may not be suitable for some highrisk patients, indicating that further research is required to reduce the radiation dose to adjacent normal tissues.

\section{CONFLICT OF INTEREST}

The authors declare no conflict of interest.

\section{REFERENCES}

Al-Sarraf M, LeBlanc M, Giri PG, Fu KK, Cooper J, Vuong T, Forastiere AA, Adams G, Sakr WA, Schuller DE, Ensley JF (1998) Chemoradiotherapy versus radiotherapy in patients with advanced nasopharyngeal cancer: phase III randomized Intergroup study 0099. J Clin Oncol 16: 1310-1317.

Chow E, Payne D, O’Sullivan B, Pintilie M, Liu FF, Waldron J, Warde P, Cummings B (2002) Radiotherapy alone in patients with advanced nasopharyngeal cancer: comparison with an intergroup study Is combined modality treatment really necessary? Radiother Oncol 63: 269-274.

Chua DT, Sham JS, Hung KN, Leung LH, Au GK (2006) Predictive factors of tumor control and survival after radiosurgery for local failures of nasopharyngeal carcinoma. Int J Radiat Oncol Biol Phys 66: 1415-1421. 
Chua DT, Sham JS, Leung LH, Au GK (2005) Re-irradiation of nasopharyngeal carcinoma with intensity-modulated radiotherapy. Radiother Oncol 77: 290-294.

Corry J, Fisher R, Rischin D, Peters LJ (2006) Relapse patterns in WHO 2/3 nasopharyngeal cancer: is there a difference between ethnic Asian vs non-Asian patients? Int J Radiat Oncol Biol Phys 64: 63-71.

Han F, Zhao C, Huang SM, Lu LX, Huang Y, Deng XW, Mai WY, Teh BS, Butler EB, Lu TX (2012) Long-term outcomes and prognostic factors of re-irradiation for locally recurrent nasopharyngeal carcinoma using intensity-modulated radiotherapy. Clin Oncol (R Coll Radiol) 24: 569-576.

Hsiung CY, Yorke ED, Chui CS, Hunt MA, Ling CC, Huang EY, Wang CJ, Chen HC, Yeh SA, Hsu HC, Amols HI (2002) Intensity-modulated radiotherapy versus conventional three-dimensional conformal radiotherapy for boost or salvage treatment of nasopharyngeal carcinoma. Int J Radiat Oncol Biol Phys 53: 638-647.

Hsu MM, Hong RL, Ting LL, Ko JY, Sheen TS, Lou PJ (2001) Factors affecting the overall survival after salvage surgery in patients with recurrent nasopharyngeal carcinoma at the primary site: experience with 60 cases. Arch Otolaryngol Head Neck Surg 127: 798-802.

Hua YJ, Chen MY, Qian CN, Hong MH, Zhao C, Guo L, Guo X, Cao KJ (2009) Postradiation nasopharyngeal necrosis in the patients with nasopharyngeal carcinoma. Head Neck 31: 807-812.

Hua YJ, Han F, Lu LX, Mai HQ, Guo X, Hong MH, Lu TX, Zhao C (2012) Long-term treatment outcome of recurrent nasopharyngeal carcinoma treated with salvage intensity modulated radiotherapy. Eur J Cancer 48: 3422-3428.

Kam MK, Teo PM, Chau RM, Cheung KY, Choi PH, Kwan WH, Leung SF, Zee B, Chan AT (2004) Treatment of nasopharyngeal carcinoma with intensity-modulated radiotherapy: the Hong Kong experience. Int J Radiat Oncol Biol Phys 60: 1440-1450.

Law SC, Lam WK, Ng MF, Au SK, Mak WT, Lau WH (2002) Reirradiation of nasopharyngeal carcinoma with intracavitary mold brachytherapy: an effective means of local salvage. Int J Radiat Oncol Biol Phys 54: 1095-1113.

Lee AW, Foo W, Law SC, Peters LJ, Poon YF, Chappell R, Sze WM, Tung SY, Lau WH, Ho JH (2000) Total biological effect on late reactive tissues following reirradiation for recurrent nasopharyngeal carcinoma. Int J Radiat Oncol Biol Phys 46: 865-872.

Lee AW, Law SC, Foo W, Poon YF, Cheung FK, Chan DK, Tung SY, Thaw M, Ho JH (1993) Retrospective analysis of patients with nasopharyngeal carcinoma treated during 1976-1985: survival after local recurrence. Int J Radiat Oncol Biol Phys 26: 773-782.

Lee AW, Sze WM, Au JS, Leung SF, Leung TW, Chua DT, Zee BC, Law SC, Teo PM, Tung SY, Kwong DL, Lau WH (2005) Treatment results for nasopharyngeal carcinoma in the modern era: the Hong Kong experience. Int J Radiat Oncol Biol Phys 61: 1107-1116.

Lu TX, Mai WY, Teh BS, Zhao C, Han F, Huang Y, Deng XW, Lu LX, Huang SM, Zeng ZF, Lin CG, Lu HH, Chiu JK, Carpenter LS, Grant WR, Woo SY, Cui NJ, Butler EB (2004) Initial experience using intensity-modulated radiotherapy for recurrent nasopharyngeal carcinoma. Int J Radiat Oncol Biol Phys 58: 682-687.

Marx RE (1983) Osteoradionecrosis: a new concept of its pathophysiology. J Oral Maxillofac Surg 41: 283-288.

Oksuz DC, Meral G, Uzel O, Cagatay P, Turkan S (2004) Reirradiation for locally recurrent nasopharyngeal carcinoma: treatment results and prognostic factors. Int J Radiat Oncol Biol Phys 60: 388-394.

Pignon JP, le Maitre A, Maillard E, Bourhis J (2009) Meta-analysis of chemotherapy in head and neck cancer (MACH-NC): an update on 93 randomised trials and 17346 patients. Radiother Oncol 92: 4-14.

Qiu S, Lin S, Tham IW, Pan J, Lu J, Lu JJ (2012) Intensity-modulated radiation therapy in the salvage of locally recurrent nasopharyngeal carcinoma. Int J Radiat Oncol Biol Phys 83: 676-683.

Roeder F, Zwicker F, Saleh-Ebrahimi L, Timke C, Thieke C, Bischof M, Debus J, Huber PE (2011) Intensity modulated or fractionated stereotactic reirradiation in patients with recurrent nasopharyngeal cancer. Radiat Oncol 6: 22.

Teo PM, Kwan WH, Chan AT, Lee WY, King WW, Mok CO (1998) How successful is high-dose ( $>$ or $=60 \mathrm{~Gy}$ ) reirradiation using mainly external beams in salvaging local failures of nasopharyngeal carcinoma? Int J Radiat Oncol Biol Phys 40: 897-913.

Wee J, Tan EH, Tai BC, Wong HB, Leong SS, Tan T, Chua ET, Yang E, Lee KM, Fong KW, Tan HS, Lee KS, Loong S, Sethi V, Chua EJ, Machin D (2005) Randomized trial of radiotherapy versus concurrent chemoradiotherapy followed by adjuvant chemotherapy in patients with American Joint Committee on Cancer/International Union against cancer stage III and IV nasopharyngeal cancer of the endemic variety. J Clin Oncol 23: 6730-6738.

Wei WI (2000) Salvage surgery for recurrent primary nasopharyngeal carcinoma. Crit Rev Oncol Hematol 33: 91-98.

Wei WI, Sham JS (2005) Nasopharyngeal carcinoma. Lancet 365: 2041-2054. Yu KH, Leung SF, Tung SY, Zee B, Chua DT, Sze WM, Law SC, Kam MK, Leung TW, Sham JS, Lee AW, Au JS, Hui EP, Sze WK, Cheng AC, Yau TK, Ngan RK, Wong FC, Au GK, Chan AT (2005) Survival outcome of patients with nasopharyngeal carcinoma with first local failure: a study by the Hong Kong Nasopharyngeal Carcinoma Study Group. Head Neck 27: 397-405.

Yu MC, Yuan JM (2002) Epidemiology of nasopharyngeal carcinoma. Semin Cancer Biol 12: 421-429.

Zheng XK, Ma J, Chen LH, Xia YF, Shi YS (2005) Dosimetric and clinical results of three-dimensional conformal radiotherapy for locally recurrent nasopharyngeal carcinoma. Radiother Oncol 75: 197-203.

This work is published under the standard license to publish agreement. After 12 months the work will become freely available and the license terms will switch to a Creative Commons AttributionNonCommercial-Share Alike 3.0 Unported License. 